\title{
Access to post - stroke physical rehabilitation after acute reperfusion therapy- the neglected link in ischemic stroke management: a retrospective cohort study
}

\section{RADU Răzvan Alexandrư ${ }^{1,2}$, TERECOASĂ Elena Oana ${ }^{2,3}$, CĂȘARU Bogdan ${ }^{3}$, ENACHE Iulian ${ }^{3}$, GHIȚĂ Cristina ${ }^{3}$, TIU Cristina ${ }^{2,3}$}

(cc) BY-NC-ND Balneo and PRM Research Journal DOI: http://dx.doi.org/10.12680/balne0.2021.418 Vol.12, No.1, March 2021 p: 46-52

Corresponding author: TERECOASĂ Elena Oana, E-mail: oana ter@yahoo.com

1. Department of Interventional Radiology, University Emergency Hospital Bucharest, Bucharest, Romania

2. "Carol Davila" University of Medicine and Pharmacy, Bucharest, Romania

3. Stroke Unit, Department of Neurology, University Emergency Hospital Bucharest, Bucharest, Romania

\begin{abstract}
Background: The burden of stroke is high in Romania and data regarding access to post - stroke rehabilitation are almost nonexistent. We aimed to determine the percentage of patients who benefited from post-stroke rehabilitation and to describe the most common rehabilitation settings.

Methods: A structured telephone-based questionnaire regarding access to post-stroke rehabilitation therapy and outcomes was administered to all patients with ischemic stroke who benefited from reperfusion therapy in a tertiary center in 2019.

Results: 211 stroke patients received reperfusion therapy during the studied period. Out of these, 208 patients were included in the initial analysis and 109 patients were deemed eligible for post-stroke rehabilitation therapy. 57 patients (55.8\%) performed poststroke rehabilitation. In-hospital rehabilitation was reported by 35 patients $(32.1 \%)$ with a median length of hospital stay of 14 days. 28 patients $(25.6 \%)$ performed home based physical therapy with a median frequency of 3 sessions per week. 12 patients $(11.1 \%)$ were admitted to nursing homes. Compared to stroke patients who did not perform in-hospital rehabilitation, those who did were younger (median age 65 years vs. 73 years, $\mathrm{p}=0.01$ ) and more likely to have moderate-severe post-stroke disability (mRS score $3-5$ at discharge $80 \%$ vs. $59.4 \%, \mathrm{p}=0.03$ ). $\mathrm{mRS}$ score at discharge $\leq 2$ was a significant predictor for not pursuing post-stroke rehabilitation $(\mathrm{p}<0.001)$.

Conclusion: Approximately 50\% of the stroke patients treated with reperfusion therapies were eligible for post-stroke rehabilitation and approximately $50 \%$ of them had access to rehabilitation therapy while only $30 \%$ had access to in-hospital rehabilitation.
\end{abstract}

Keywords: Stroke rehabilitation; Eastern Europe; Romania; Rehabilitation Center; Physical Therapy,

\section{Introduction}

As an overwhelming disease of huge medical, social and economic significance, stroke is now the second leading cause of disability and death worldwide.(1) Eastern Europe, where Romania is located, is the region with the second highest stroke incidence in the world, after east Asia, being estimated that around $30 \%$ of the adult population is at risk of suffering a stroke during their lifetime.(2,3) Despite this high stroke burden, this region reports overall low access to appropriate acute and post acute stroke care and reperfusion therapies.(4) In order to offset the dramatic impact that a future increase in stroke prevalence is expected to have in Europe, a Stroke Action Plan for Europe (SAP-E) was recently developed. This paper emphasizes the importance of national stroke plans that should encompass an entire "stroke chain", from primary prevention to life after stroke and also offers targets and guidance for every link in this chain.(5)

In order to provide equal access to proven effective therapies for all stroke patients, healthcare policy makers, public healthcare specialists and clinicians across the world should first analyse their existing individual capabilities and unique infrastructure. Assessment of the existing status is a key reference point that should lead to the settlement of an interconnected stroke network rather than myopic singular stroke ready hospitals.(6) This implies focusing on every link in the stroke care chain starting from stroke awareness and ending with comprehensive rehabilitation and adequate post-stroke care.(5)

The 2006 Helsingborg declaration stated that all stroke patients in Europe should have access to appropriate rehabilitation by 2015.(7) However, in 2017 access to post - stroke inpatient rehabilitation was found to vary widely, the percentages of those actually benefiting from this therapy ranging from 15.1 to $44.3 \%$ in different European countries.(8) Access to post - stroke rehabilitation is expected to be low in Eastern Europe but available high - quality data on this subject are scarce or even non - existent for most of the countries from this region. Available published data shows unequal and scant availability of rehabilitation services. Moreover, post stroke rehabilitation in Eastern Europe is usually focused 
only on physical therapy, while access to occupational therapy, speech therapy and psychological care is generally lacking.(9)

The post - stroke care costs as well as the effectiveness of rehabilitation are dependent on the quality of rehabilitation services available for each stroke patient. Early supported discharge services with multidisciplinary coordination reduce in-hospital stay, long-term dependency and increase the proportion of stroke patients living at home.(10) European National healthcare systems should strive to adhere to SAP-E recommendations, aiming to build adequate post - stroke rehabilitation systems capable of providing: (1) access to appropriate rehabilitation for $90 \%$ of stroke patients and (2) early supported discharge for at least $20 \%$ of stroke patients. Data concerning post - stroke access to rehabilitation across Eastern European countries are currently collected by the Registry of Stroke Quality (RES - Q) endorsed by the European Stroke Organisation - East Programme.(11) However, by capturing mainly data regarding access to early supported discharge, this registry underreports overall access to post - stroke rehabilitation as waiting times between initial hospital discharge and admission in a Department of Rehabilitation or first evaluation by a specialist in rehabilitation therapy can be rather long in many Eastern European countries.(12)

Romania provides publicly - funded healthcare to all citizens, including hospitalization for rehabilitation therapy. Post - acute in - hospital rehabilitation for stroke patients takes place in designated wards or hospitals after discharge from a Stroke Unit or Department of Neurology. Public nursing homes are scarce and therefore patients are usually admitted to private nursing homes. In an attempt to support the long - term care of stroke survivors, the Romanian authorities offer a monthly allowance on the basis of the degree of disability which can be used to partially cover the expenses of private nursing homes or rehabilitation in private settings.

The aim of this study was to determine the access to post - stroke rehabilitation therapy and to describe the setting of rehabilitation in a population of patients with acute ischemic stroke treated by intravenous thrombolysis and/or mechanical thrombectomy in the University Emergency Hospital Bucharest.

\section{Materials and Methods}

All stroke patients treated by intravenous thrombolysis (IVT) and/or endovascular procedures (EVT) in the University Emergency Hospital Bucharest between 01.01.2019 and 31.12.2019 were screened for eligibility to be included in this study. Informed consent for scientific and clinical studies was signed by the patients and/or their caregivers at admission and all patients were included in the local database of the Romanian Registry for Interventional Treatment in Acute Stroke (RRIT-AS). This database includes prospectively collected data on all ischemic stroke patients who receive reperfusion therapy at admission. Patients were excluded from the study if: (1) they were discharged with a modified Rankin score $(\mathrm{mRS})$ of 0 or 6; (2) registry data was incomplete (3) they or their caregivers refused to participate in this study during the telephone interview (4) they or their caregivers were unreachable by phone. The flow diagram of the study population is represented in Figure 1.

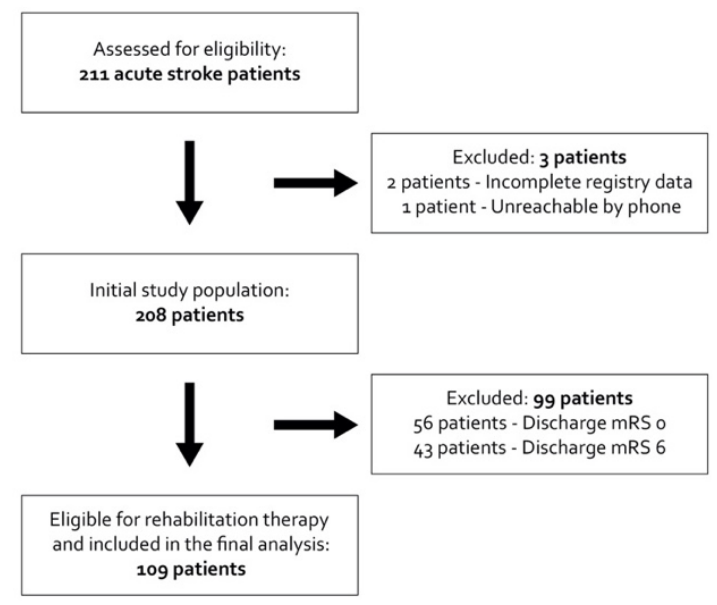

Figure 1. Diagram of the study population.

A structured questionnaire was developed and administered to patients or their caregivers by telephone interview by two experienced stroke neurology residents B.C and I.E between 01.07.2020 and 15.07.2020. The questionnaire aimed to answer the following questions: (1) Which was the mRS score at the time of telephone contact?; (2) Was the patient living at home or in a nursing home?; (3) Did the patient benefit from rehabilitation services provided by a rehabilitation institution after their discharge?; (4) If the patient benefited from rehabilitation in an institution, was there any delay between discharge from the Neurology Department and admittance to the rehabilitation institution?; (5) If the patient benefited from rehabilitation in an institution, how many times was the patient hospitalized in the institution?; (6) If the patient benefited from rehabilitation in an institution, which was the length of hospital stay there?; (7) If the patient benefited from rehabilitation in an institution, was the patient hospitalized in a private or public rehabilitation institution?; (8) If the patient didn't benefit from rehabilitation, which was the reason?; (9) Did the patient benefit from rehabilitation at home?; (10) If the patient benefited from rehabilitation at home, was it supported by public health insurance or by personal funding?; (11) How many times a week did the patient receive rehabilitation services at home?; (12) Was the patient granted a degree of disability and corresponding allowance?; (13) Did the patient receive an attendant allowance? The simplified mRS questionnaire was 
translated into Romanian and used as the reference questionnaire for obtaining the $\mathrm{mRS}$ score of the patients.(13) Access to rehabilitation was defined as access to any kind of rehabilitation. Rehabilitation institutions were considered all public or private hospitals providing rehabilitation services as in-patient care. The mean number of days spent in the rehabilitation institution and number of admissions were recorded separately during the interview for each patient.

Clinical data and demographic data were extracted from the local database of the RRIT-AS and linked to the survey data. Initial stroke severity was classified according to the National Institute of Health Stroke Scale (NIHSS). Short - term stroke outcome was defined as outcome at the moment of hospital discharge and was assessed with the mRS score. Patients with mRS scores 0 - 2 were considered functionally independent. Both mRS and NIHSS scores were assigned by attending physicians during hospitalization and included in the Stroke Registry after patient discharge. Traditional risk factors analysed in this study were defined as follows: (1) diabetes mellitus: clinical history of diabetes mellitus or glycated haemoglobin $\geq 6.5 \%$ or random plasma glucose of $\geq$ $200 \mathrm{mg} / \mathrm{dl}$ or fasting glucose level $\geq 126 \mathrm{mg} / \mathrm{dl}$; (2) dyslipidaemia: low density lipoprotein cholesterol $\geq$ $100 \mathrm{mg} / \mathrm{dL}$ or triglycerides $\geq 150 \mathrm{mg} / \mathrm{dl}$ or previous treatment with a statin; (3) arterial hypertension: history of systolic blood pressure $\geq 140 \mathrm{mmHg}$ and/or diastolic blood pressure $\geq 90 \mathrm{mmHg}$ on two separate occasions or persistently elevated blood pressure during hospitalization or prior antihypertensive treatment; (4) coexistent cardiovascular disease: history of ischemic heart disease and/or diagnosis of ischemic heart disease and/or history or diagnosis of peripheral artery disease; (5) previous stroke: clinical history of stroke (silent lesions found on CT were not taken into consideration). (6) major cognitive decline: history of pre - stroke dementia. Stroke aetiology was assessed in accordance with Trial of Org 10172 in Acute Stroke Treatment (TOAST) criteria.(14)

All statistical analyses were performed using NCSS 12 Statistical Software (NCSS, LLC. Kaysville Utah, USA) and Medcalc Statistical Software 18.11.3 (Medcalc Software, Ostend, Belgium). Statistical analysis was limited to bivariate analysis due to the small sample size. A pre-set significance level of $\mathrm{p}<0.05$ was considered statistically significant for all comparisons. Continuous variables are described as mean $+/-$ standard deviations (SD) or median and 25 - 75 IQR and categorical variables as absolute numbers and percentages. For comparison between continuous variables, Mann Whitney or Kruskal Wallis test were used, according to the number of selected variables. Chi-squared test and Fisher's exact test were used for testing the strength of association between categorical variables.

\section{Results}

A total number of 211 stroke patients were treated by IVT/EVT in 2019 in our hospital. 3 patients had incomplete registry data or were unreachable for the telephone interview so that the initial study population included 208 patients. Since 99 of the 208 patients had discharge mRS scores 0 or 6 and were considered non eligible for rehabilitation therapy, 109 patients were included in the final analysis of this study. The clinical and demographic characteristics of these patients are listed in Table 1

57 patients $(52.3 \%$ of the stroke patients deemed eligible for rehabilitation therapy or $27.4 \%$ of the stroke patients initially included in the study) reported to have performed any kind of rehabilitation (including: inhospital rehabilitation, outpatient rehabilitation, physical rehabilitation at home, physical rehabilitation in the nursing home). The setting of rehabilitation procedures is detailed in Table 2.

In-hospital post - stroke rehabilitation after discharge from our department was reported by 35 patients (32.1\%), with a median length of stay per hospitalization of 14 days (Range: $7-60$ days). Out of these patients, 18 $(51.4 \%)$ were admitted for in - hospital rehabilitation one time, 7 patients $(20 \%)$ two times, 4 patients $(11.4 \%)$ three times and the remaining were admitted four or more times. $29(82.8 \%)$ of these patients benefited from rehabilitation in a public hospital, $4(11.4 \%)$ in a private hospital and $2(5.7 \%)$ opted for both private and public hospital admissions. There was no statistically significant difference between the number of in - hospital rehabilitation admissions for patients hospitalized in our department in the first or last 6 months of 2019 (mean 2.57 vs 1.81 times, $p=0.1) .29(82.8 \%)$ of the 35 patients who performed in - hospital post - stroke rehabilitation benefited from early supported discharge, while 6 $(17.2 \%)$ were admitted to the rehabilitation facility in the first weeks after the discharge from our department. 14 patients $(40 \%)$ of those who benefited from in-hospital rehabilitation continued physical therapy at home. As compared to stroke patients who did not perform in hospital rehabilitation, those who did were younger (median age 65 years vs. 73 years, $p=0.01$ ) and more frequently had moderate - severe post - stroke disability (mRS score $3-5$ at discharge $80 \%$ vs. $59.4 \%, \mathrm{p}=0.03$ ).

Physical therapy at home was reported by 28 patients $(25.6 \%)$. Out of these patients, $14(50 \%)$ performed rehabilitation only in this setting, while the others were first admitted for in - hospital rehabilitation and then continued rehabilitation therapy at home. The median period of performing rehabilitation procedures at home was 3 months (25 - 75 IQR: $2-7.7$ months) and the median frequency of sessions was 3 times per week (2575 IQR: 2 - 4 times/week). Only 7 patients (25\%) of those who reported physiotherapy at home applied for 
and received public reimbursement of the home - based physical therapy.

Out of the 109 patients included in the final analysis, 12 (11.1\%) were admitted to a nursing home at any time after discharge from our department. All nursing homes were private facilities and caregivers reported that they offered at least physical therapy to their patients.

66 patients $(60.5 \%)$ did not undergo in-hospital rehabilitation and were not admitted to nursing homes. The most frequently reported reasons for not accessing inpatient rehabilitation were: 1) patients thought they don't need it (31 patients, 46.9\%), 2) patients didn't want to go to rehabilitation (11 patients,16.6\%), 3) patients didn't know that they should go to rehabilitation (6 patients, 9.1\%). Other less frequently mentioned reasons for not pursuing rehabilitation were: couldn't afford rehabilitation, were not suitable candidates for rehabilitation due to other severe medical illnesses.

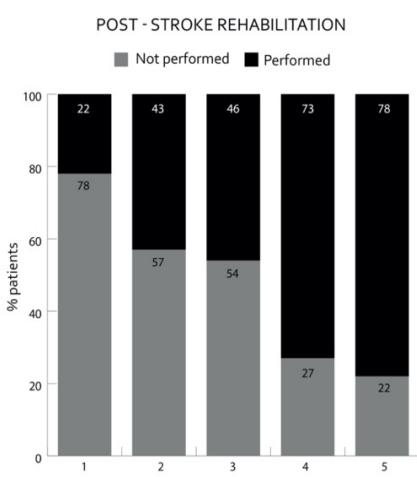

mRS score at discharge

Figure 2. Access to post stroke rehabilitation in the study population

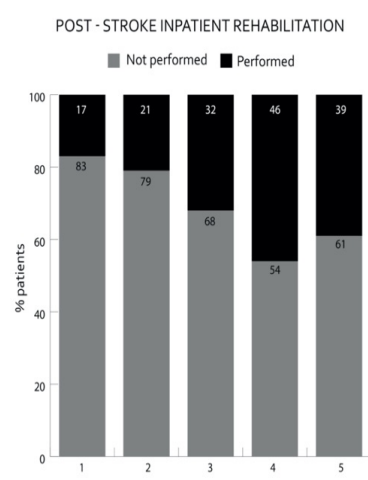

Figure 3. Access to in-patient rehabilitation in the study population
A low mRS score at discharge was a significant predictor for not pursuing post-stroke rehabilitation $(\mathrm{p}<0.001) .37$ patients $(33.9 \%)$ were discharged with an mRS score of 1 or 2 points. 7 of them $(18.9 \%)$ reported having benefited from in-hospital post-stroke rehabilitation and 3 of them (8.1\%) from physical therapy at home, while the other 26 (70.2\%) did not pursue post - stroke rehabilitation in any setting. The most frequently reported reasons for not pursuing rehabilitation in patients discharged with $\mathrm{mRS}$ scores $1-2$ were: 1) considered it was not needed $(90.91 \%)$ and 2$)$ didn't want to perform it $(4.55 \%) .72$ patients $(66.1 \%)$ were discharged with $\mathrm{mRS}$ scores $3-5$. 28 of these patients (38.9\%) performed in - hospital rehabilitation, 11 (15.2\%) performed only physical procedures at home, 7 patients $(9.7 \%)$ were admitted to a nursing home, while $26(36.1 \%)$ of them did not perform post - stroke rehabilitation. These results are detailed in Figures 2 and 3.

By the time of the telephone interview, $22(20.02 \%)$ of the patients included in the final analysis died. Eleven patients $(10.1 \%)$ died after discharge without benefiting from any post-stroke rehabilitation: 4 (3.6\% of the study population) had an mRS score of 2 at discharge and reported that they did not consider rehabilitation necessary, while the remaining 7 (6.4\%) reported either that they couldn't afford it or that they didn't know that post-stroke rehabilitation was an option or they died before being admitted to rehabilitation.

Out of the 87 patients who were alive at the time of the survey, $26(29.9 \%)$ applied for and received disability allowances and $14(16.1 \%)$ received a supplementary allowance to support the payment of a personal caregiver.

\section{Discussion}

The population of this study consisted of patients with acute ischemic stroke who benefited from reperfusion therapies in accordance with national and international guidelines.(15) Stroke outcome after IVT/EVT is usually assessed at three months after the cerebrovascular event. According to different meta - analysis and trials, 43.5 $55 \%$ of patients achieve functional independence (mRS scores $0-2)$ by this time.(16-18) However, studies performed in healthcare systems with limited resources have shown that stroke patients from these countries generally have worse outcomes than those reported in landmark papers, probably due to multiple factors, including quality of acute stroke care and access to rehabilitation therapies.(19) This finding emphasizes that stroke care is a continuum and post-stroke rehabilitation should accompany acute stroke therapies.

Recent estimates for Europe suggest a $27 \%$ percent increase in the demand for rehabilitation and long - term care services after stroke by 2047 . An East - West gradient of increasing stroke burden is expected to affect European countries in the future. This gradient is most probably attributable to insufficient and ineffective strategies of primary stroke prevention and below standard quality of acute and long - term stroke care in many Eastern European countries.(20) Given the presumed low - access to adequate stroke care in the region, healthcare policymakers should work together and focus on proven disability - reducing therapies such as admittance to stroke units, thrombectomy, thrombolysis and early supported discharge to rehabilitation services.(21)

According to the Stroke Alliance For Europe (SAFE) report, the percentage of patients that have access to post stroke rehabilitation widely varies from $25 \%$ to $60 \%$ in the Eastern European countries for which data on this subject is available.(9) However, these data are frequently based on surveys among key opinion leaders and papers that are usually more than ten years old and rarely on epidemiological studies or database inquiries. Romania is listed in the SAFE report with $30 \%$ of stroke patients having access to post-stroke rehabilitation in $2005 / 2006$ and a subsequent paper regarding stroke care in Eastern Europe from 2012 states that efforts are being made to create neuro - rehabilitation units in Romania. 
$(22,23)$ Our results show that in the probably best available scenario, as most of the study population resides in Bucharest, the capital and the most developed city of the country, only $27.4 \%$ of the stroke patients included in this study benefited from this much needed therapy. The comparison of this percentage with the one reported by SAFE would lead to the conclusion that during the past 15 years access to post - stroke rehabilitation in Romania remained unchanged or has actually worsened, which definitely cannot be true.

In-hospital rehabilitation is the most standardised and well-defined type of post - stroke rehabilitation and therefore the most suitable for being analyzed in studies comparing efficacy and access to this therapy. In our study population, $21.2 \%$ of the stroke patients discharged alive had access to in - patient rehabilitation therapy, a percentage that is significantly lower than that reported by other countries. $(8,24)$

In order to improve access to rehabilitation for stroke patients and to adhere to European standards, Romania and other Eastern European countries should first focus on obtaining and analysing reliable data regarding their current status, which would probably be very similar to Brazilian estimates that more than $70 \%$ of patients do not have access to any kind of post stroke rehabilitation.(25) These data should include quantitative and qualitative parameters that would facilitate further comparisons between Eastern and Western European countries and ultimately determine clinical effectiveness. For example, a higher number of patients are being transferred directly to post stroke rehabilitation facilities in Sweden than in Latvia, which probably implies better stroke outcomes regardless of the total percentage of patients who have access to post-stroke rehabilitation. Moreover, while in Romania and Latvia patients usually spend around two weeks in rehabilitation facilities, they spend a far longer time in Sweden or in Poland, which may also impact the stroke outcome. $(12,26)$

Current recommendations state that all stroke patients should be evaluated by rehabilitation specialists in order to identify any "rehabilitation potential". However, even some high income countries, such as Australia, report that less than $50 \%$ of the patients are being evaluated for scheduling the type of post-stroke rehabilitation during their stay on stroke units.(27) In lower - income countries this percent might be far worse. In our study, a minority of patients were evaluated by a rehabilitation specialist during hospital admission. The implementation of neuro- rehabilitation wards dedicated to stroke and other specific neurological diseases could facilitate the early evaluation of this category of patients and could improve the collaboration between neurologists and rehabilitation specialists with the ultimate goal of increasing the number of stroke patients benefiting from rehabilitation therapy.
Patients and caregivers' awareness regarding post stroke rehabilitation was poor in our study population. A significant percentage of those who did not benefit from this therapy reported that they "thought they didn't need rehabilitation therapy", they "didn't know that they should undergo rehabilitation therapy" or they "didn't want to perform rehabilitation therapy". Frequently overlooked in the setting of emergency hospitals, detailed discussions between neurologists, rehabilitation specialists and stroke patients or caregivers during hospitalization for acute care might change the perspective of patients who state that they "don't want" to perform rehabilitation procedures, thereby improving stroke outcome.(28)

Our study population consisted of all stroke patients treated by IVT/EVT during one year. We considered that this population was "the best - case scenario" for our country since patients who receive reperfusion therapies tend to be younger, have more severe strokes and a higher likelihood of pre-stroke independence $(15,24)$, which makes them suitable candidates for post - stroke rehabilitation. However, the selection on the basis of this criteria is also a major limitation of our study due to the lack of external validity to a general stroke population. Nevertheless, the low number of patients benefiting from in - hospital rehabilitation in this "best case scenario", should act as an incentive to enhance cooperation between neurologists and rehabilitation specialists in order to achieve European targets regarding rehabilitation therapy designed for all stroke patients.

Another significant drawback of our study was the variable length of follow-up. A trend towards more frequent admissions for in - hospital rehabilitation could be observed for patients discharged in the first 6 months of 2019 as compared to those discharged in the last 6 months of 2019. A future study with prospective collection of data regarding post - stroke rehabilitation therapy at pre - specified time points after acute hospital care is definitely needed in Romania.

5. Conclusion In our cohort of acute ischemic stroke patients who benefited from reperfusion therapies, 51.6\% were likely candidates for post - stroke rehabilitation but only $27.4 \%$ actually accessed this much needed therapy. $16.8 \%$ of the study population benefited from post stroke rehabilitation in an inpatient setting. Approximately two thirds of our stroke patients discharged with mRS scores of $1-2$ and one third of those discharged with mRS scores of $3-5$ did not perform any type of rehabilitation therapy. Access to post - stroke rehabilitation was poor in our cohort. The real situation is probably worse throughout the country and therefore an urgent action to improve access of stroke survivors to rehabilitation therapy is needed. 


\section{Conflicts of interest and Ethics}

The authors declare no conflict of interest.

\section{Institutional Review Board Statement:}

Ethical review and approval were waived for this study, due to the fact that the National Stroke Registry was approved by the relevant Ethics Committee and patients provided written informed consent for inclusion in the National Registry and for the subsequent data use. Informed consent was obtained from all subjects or caregivers involved in the study.

\section{Author Contributions:}

Conceptualization of the study R.A.R and E. O.T; Methodology E.O.T; Data Curation: B.C, I.E, C.G, Writing - original draft: R.A.R; Writing -review and editing: R.A.R, E.O.T, C.T; Supervision. C.T; Funding acquisition: CT; All authors have read and agreed to the published version of the manuscript.

\section{References:}

1. Johnson CO, Nguyen M, Roth GA, Nichols E, Alam T, Abate D, et al. Global, regional, and national burden of stroke, 19902016: a systematic analysis for the Global Burden of Disease Study 2016. Lancet Neurol. 2019;18(5):439-58.

2. Krishnamurthi R V., Ikeda T, Feigin VL. Global, Regional and Country-Specific Burden of Ischaemic Stroke, Intracerebral Haemorrhage and Subarachnoid Haemorrhage: A Systematic Analysis of the Global Burden of Disease Study 2017. Neuroepidemiology. 2020;54(2):171-9.

3. Global, Regional, and Country-Specific Lifetime Risks of Stroke, 1990 and 2016. N Engl J Med. 2018 19;379(25):2429-37.

4. Aguiar de Sousa D, von Martial R, Abilleira S, Gattringer T, Kobayashi A, Gallofré M, et al. Access to and delivery of acute ischaemic stroke treatments: A survey of national scientific societies and stroke experts in 44 European countries. Eur Stroke J. 2019;4(1):13-28.

5. Norrving B, Barrick J, Davalos A, Dichgans M, Cordonnier C, Guekht A, et al. Action Plan for Stroke in Europe 2018-2030. Eur Stroke J. 2018 Oct 29;3(4):309-36.

6. Vivanco-Hidalgo RM, Abilleira S, Salvat-Plana M, Ribera A, Gallofré G, Gallofré M. Innovation in systems of care in acute phase of ischemic stroke. The experience of the catalan stroke programme. Front Neurol. 2018;9:1-6.

7. Kjellström T, Norrving B, Shatchkute A. Helsingborg declaration 2006 on European Stroke Strategies. Cerebrovasc Dis. 2007;23(2-3):229-41.

8. Lynch EA, Cadilhac DA, Luker JA, Hillier SL. Inequities in access to inpatient rehabilitation after stroke: An international scoping review. Top Stroke Rehabil. 2017;24(8):619-26.

9. Stevens E, McKevitt C, Emmett E, Wolfe CDA, Wang Y, et al. Stroke Alliance for Europe. The Burden of Stroke in Europe Report (2017). London: Stroke Alliance for Europe. Available at: https://strokeeurope.eu/media/download/ (Accessed 14 August 2020)

10. P L, S B, Early Supported Discharge Trialists. Early supported discharge services for people with acute stroke (Cochrane review). Cochrane Database Syst Rev 2017. 2017;(7):CD000443.

11. Mikulík R, Caso V, Bornstein NM, Svobodová V, Pezzella FR, Grecu A, et al. Enhancing and accelerating stroke treatment in Eastern European region: Methods and achievement of the ESO EAST program. Eur Stroke J. 2020;5(2):204-12.

12. Opara JA, Langhorne P, Larsen T, Mehlich K, Szczygiel J.
Facilities of early rehabilitation after stroke in Poland 2010. Int J Rehabil Res. 2012;35(4):367-71.

13. Bruno A, Akinwuntan AE, Lin C, Close B, Davis K, Baute V, et al. Simplified modified rankin scale questionnaire: Reproducibility over the telephone and validation with quality of life. Stroke. 2011;42(8):2276-9.

14. Adams HP, Bendixen BH, Kappelle LJ, Biller J, Love BB, Gordon DL, et al. Classification of subtype of acute ischemic stroke. Stroke. 1993;24(1):35-41.

15. Powers WJ, Rabinstein AA, Ackerson T, Adeoye OM, Bambakidis NC, Becker K, et al. Guidelines for the early management of patients with acute ischemic stroke: 2019 update to the 2018 guidelines for the early management of acute ischemic stroke a guideline for healthcare professionals from the American Heart Association/American Stroke A. Vol. 50, Stroke. 2019. 344-418.

16. Emberson J, Lees KR, Lyden P, Blackwell L, Albers G, Bluhmki E, et al. Effect of treatment delay, age, and stroke severity on the effects of intravenous thrombolysis with alteplase for acute ischaemic stroke: A meta-analysis of individual patient data from randomised trials. Lancet. 2014;384(9958):1929-35.

17. Goyal M, Menon BK, Van Zwam WH, Dippel DWJ, Mitchell PJ, Demchuk AM, et al. Endovascular thrombectomy after largevessel ischaemic stroke: A meta-analysis of individual patient data from five randomised trials. Lancet.2016;387(10029):172331.

18. Wahlgren N, Ahmed N, Dávalos A, Ford GA, Grond M, Hacke $\mathrm{W}$, et al. Thrombolysis with alteplase for acute ischaemic stroke in the Safe Implementation of Thrombolysis in StrokeMonitoring Study (SITS-MOST): an observational study. Lancet. 2007, 27;369(9558):275-82.

19. Martins SO, Mont'Alverne F, Rebello LC, Abud DG, Silva GS, Lima FO, et al. Thrombectomy for stroke in the public health care system of Brazil. N Engl J Med. 2020;382(24):2316-26.

20. Wafa HA, Wolfe CDA, Emmett E, Roth GA, Johnson CO, Wang Y. Burden of Stroke in Europe: Thirty-Year Projections of Incidence, Prevalence, Deaths, and Disability-Adjusted Life Years. Stroke. 2020

21. Campbell BCV, Mitchell PJ, Churilov L, Keshtkaran M, Hong $\mathrm{KS}$, Kleinig TJ, et al. Endovascular thrombectomy for ischemic stroke increases disability-free survival, quality of life, and life expectancy and reduces cost. Front Neurol. 2017;8.:1-7.

22. Czlonkowska A, Skowroñska M, Niewada M. Stroke service in Central and Eastern Europe. Int J Stroke. 2007;2(4):276-8

23. Lenti L, Brainin M, Titianova E, Morovic S, Demarin V, Kalvach P, et al. Stroke care in Central Eastern Europe: Current problems and call for action. Int J Stroke. 2013;8(5):365-71

24. Labberton AS, Barra M, Rønning OM, Thommessen B, Churilov L, Cadilhac DA, et al. Patient and service factors associated with referral and admission to inpatient rehabilitation after the acute phase of stroke in Australia and Norway. BMC Health Serv Res. 2019;19(1):871.

25. Silva GS, Rocha ECA, Pontes-Neto OM, Martins SO. Stroke Care Services in Brazil. J Stroke Med. 2018;1(1):51-4.

26. Bērziña G, Vētra A, Sunnerhagen KS. A comparison of stroke rehabilitation; data from two national cohorts. Acta Neurol Scand. 2016;134(4):284-91.

27. Lynch EA, Luker JA, Cadilhac DA, Hillier SL. Inequities in access to rehabilitation: Exploring how acute stroke unit clinicians decide who to refer to rehabilitation.Disabil Rehabil.2016;38(14):1415-24.

28. Koh W, Barr C, George S. After Discharge From Hospital. 2011;187(11):799-804. 
Table 1. Clinical and demographic characteristics of the study population

\begin{tabular}{|c|c|c|c|c|}
\hline & $\begin{array}{l}\text { Overall } \\
(n=109)\end{array}$ & $\begin{array}{l}\text { Performing post }- \text { stroke } \\
\text { rehabilitation } \\
(\mathrm{n}=57)\end{array}$ & $\begin{array}{l}\text { Not performing post }- \text { stroke } \\
\text { rehabilitation } \\
(n=52)\end{array}$ & p - value \\
\hline \multicolumn{5}{|l|}{ Demographics } \\
\hline Age (years), median & $71(62-79)$ & $70(57-78)$ & $72(63-80)$ & 0.1 \\
\hline Female Sex, \% & $56(51.4 \%)$ & $30(52.6 \%)$ & $26(50 \%)$ & 0.7 \\
\hline Urban area, \% & $81(74.3 \%)$ & $46(80.7 \%)$ & $35(67.3 \%)$ & 0.1 \\
\hline \multicolumn{5}{|l|}{ Comorbidities \& Risk Factors } \\
\hline Arterial Hypertension, $\%$ & $67(61.5 \%)$ & $34(59.6 \%)$ & $33(63.5 \%)$ & 0.6 \\
\hline Dyslipidaemia, \% & $65(59.6 \%)$ & $36(63.2 \%)$ & $29(55.7 \%)$ & 0.4 \\
\hline $\mathrm{CV}$ disease, $\%$ & $15(13.7 \%)$ & $10(17.5 \%)$ & $5(9.6 \%)$ & 0.6 \\
\hline Diabetes mellitus, $\%$ & $23(21.1 \%)$ & $9(15.8 \%)$ & $14(26.9 \%)$ & 0.1 \\
\hline Atrial fibrillation, $\%$ & $23(21.1 \%)$ & $12(21.1 \%)$ & $11(21.2 \%)$ & 0.9 \\
\hline Prior stroke, $\%$ & $17(15.6 \%)$ & $7(12.3 \%)$ & $10(19.2 \%)$ & 0.3 \\
\hline Prior major cognitive decline, $\%$ & $2(1.8 \%)$ & 0 & $2(3.8 \%)$ & 0.1 \\
\hline \multicolumn{5}{|l|}{ Stroke - related characteristics } \\
\hline Stroke territory & & & & 0.9 \\
\hline Left carotid & $57(52.3 \%)$ & $29(50.9 \%)$ & $28(53.8 \%)$ & \\
\hline Right carotid & $39(35.8 \%)$ & $21(36.8 \%)$ & $18(34.6 \%)$ & \\
\hline Vertebro-basilar & $13(11.9 \%)$ & $7(12.3 \%)$ & $6(11.5 \%)$ & \\
\hline Admission NIHSS, median & $16(8-21)$ & $17(10-22)$ & $11(6.2-9.7)$ & 0.008 \\
\hline Initial stroke severity & & & & 0.01 \\
\hline Mild stroke $<8$ & $25(22.9 \%)$ & $8(14 \%)$ & $17(32.7 \%)$ & \\
\hline Moderate stroke 8-16 & $37(33.9 \%)$ & $18(31.6 \%)$ & $19(36.5 \%)$ & \\
\hline Severe stroke $>16$ & $47(43.2 \%)$ & $31(54.4 \%)$ & $16(30.8 \%)$ & \\
\hline Discharge NIHSS, median & $5(2-13)$ & $9(3.5-4.5)$ & $3(1-6)$ & $<0.001$ \\
\hline Stroke severity at discharge & & & & $<0.001$ \\
\hline Mild stroke $<8$ & $65(59.6 \%)$ & $22(38.6 \%)$ & $43(82.7 \%)$ & \\
\hline Moderate stroke 8-16 & $30(27.5 \%)$ & $26(45.6 \%)$ & $4(7.7 \%)$ & \\
\hline Severe stroke $>16$ & $14(12.9 \%)$ & $9(15.8 \%)$ & $5(9.6 \%)$ & \\
\hline Discharge mRS, median & $3(2-4)$ & $4(3-4.5)$ & $2.5(1-3)$ & $<0.001$ \\
\hline Discharge $\mathrm{mRS}, \%$ & & & & $<\mathbf{0 . 0 0 1}$ \\
\hline mRS scores $1-2$ & $37(33.9 \%)$ & $11(19.3 \%)$ & $26(50 \%)$ & \\
\hline mRS scores $3-5$ & $72(66.1 \%)$ & $46(80.7 \%)$ & $26(50 \%)$ & \\
\hline Follow-up mRS, mean \pm SD & $3(1-5)$ & $3(2-5)$ & $2(1-3.7)$ & 0.009 \\
\hline Follow-up mRS categories, $\%$ & & & & 0.07 \\
\hline mRS scores $0-2$ & $46(42.2 \%)$ & $19(33.3 \%)$ & $27(51.9 \%)$ & \\
\hline mRS scores 3-5 & $41(37.6 \%)$ & $27(47.4 \%)$ & $14(26.9 \%)$ & \\
\hline mRS score 6 & $22(20.2 \%)$ & $11(19.3 \%)$ & $11(21.1 \%)$ & \\
\hline Thrombolysis, $\%$ & $88(80.7 \%)$ & $45(80.3 \%)$ & $43(82.7 \%)$ & 0.7 \\
\hline Thrombectomy, \% & $29(26.6 \%)$ & $21(36.8 \%)$ & $8(15.4 \%)$ & 0.01 \\
\hline Stroke etiology by TOAST criteria & & & & 0.7 \\
\hline Cardioembolic & $48(44.1 \%)$ & $26(45.6 \%)$ & $22(43.1 \%)$ & \\
\hline Large artery disease & $24(22 \%)$ & $12(21.1 \%)$ & $12(23.5 \%)$ & \\
\hline Small vessel disease & - & - & - & \\
\hline Other etiology & $2(1.8 \%)$ & $1(1.7 \%)$ & $1(1.9 \%)$ & \\
\hline Undetermined etiology & $35(32.1 \%)$ & $18(31.5 \%)$ & $17(33.33 \%)$ & \\
\hline
\end{tabular}

Table 2. Setting of rehabilitation therapy for the study population ( $\mathrm{n}=109$ patients)

\begin{tabular}{lcc}
\hline Type of rehabilitation & Number of patients & $\mathbf{\%}$ \\
\hline Only physical therapy at home & 14 & 12.8 \\
Only in - hospital rehabilitation & 18 & 16.5 \\
In-hospital rehabilitation + physical therapy at home & 13 & 11.9 \\
In-hospital rehabilitation + nursing home & 3 & 2.7 \\
In-hospital rehabilitation + nursing home + physical therapy at home & 1 & 0.9 \\
Nursing home + physical therapy at home & 0 & - \\
Only nursing home & 8 & 7.3 \\
\hline Total benefiting from physical therapy at home at any time post - discharge & $\mathbf{2 8}$ & $\mathbf{2 5 . 6}$ \\
Total benefiting from in-hospital rehabilitation & $\mathbf{3 5}$ & $\mathbf{3 2 . 1}$ \\
Total benefiting from admission in a nursing home & $\mathbf{1 1}$ & $\mathbf{1 1}$ \\
\hline
\end{tabular}

